Y. Morimoto

Nagoya Math. J.

Vol. 101 (1986), 131-150

\title{
PROPAGATION OF WAVE FRONT SET IN GEVREY CLASS FOR AN EXAMPLE OF HYPERBOLIC SYSTEM
}

\author{
YOSHINORI MORIMOTO \\ Dedicated to the memory of Professor Hitoshi Kumano-go
}

\section{Introduction}

In this paper we show, for an example of hyperbolic system, that the wave front set in Gevrey class of a certain solution propagates precisely along "generalized broken null-bicharacteristic flow" defined by Kumanogo-Taniguchi [7] and Wakabayashi [10] [11].

Let $L$ be a hyperbolic system of the form

$$
\left(\begin{array}{cc}
p_{1}\left(t, x, D_{t}, D_{x}\right) & 0 \\
0 & p_{2}\left(t, x, D_{t}, D_{x}\right)
\end{array}\right)+\left(\begin{array}{ll}
0 & 1 \\
1 & 0
\end{array}\right) \quad \text { in } R_{t}^{1} \times R_{x}^{4},
$$

where $p_{j}=D_{t}+\lambda_{j}\left(t, x, D_{x}\right), j=1,2$, and

$$
\left\{\begin{array}{l}
\lambda_{1}(t, x, \xi)=0 \\
\lambda_{2}(t, x, \xi)=\xi_{1}+x_{1} \xi_{2}+x_{2} \xi_{3}+\left(t^{4}-8 x_{1} t^{3}+48 x_{2} t^{2}-192 x_{3} t\right) \xi_{4} .
\end{array}\right.
$$

We consider the Cauchy problem

$$
L U=0, \quad U(0, x)=G, \quad G \in \mathscr{E}^{\prime} .
$$

Let $\tilde{\rho}_{0}$ be a point $(0 ;(0,0,0,0,1))$ in the cotangent space $T^{*}\left(R_{t} \times R_{x}^{4}\right)$ and let $\tilde{C}_{0}$ be an integral curve in $T^{*}\left(R_{t} \times R_{x}^{4}\right)$ through $\tilde{\rho}_{0}$ by Hamilton vector field $H_{p_{1}+p_{2}}$. For $\tilde{\rho}_{0} \in T^{*}\left(R_{t} \times R_{x}^{4}\right)$ we put $\rho_{0}=\left(0, \eta^{0}\right) \in T^{*} R_{x}^{4}, \eta^{0}=(0,0,0,1)$ $\in R_{\xi}^{4} \backslash 0$. Assume that the initial value $G$ of (C.P) satisfies

$$
W F_{\kappa} G=\left\{c \rho_{0} \equiv\left\{\left(0, c \eta^{0}\right) ; c>0\right\}, 1<\kappa<3\right\},
$$

where we denote by $W F_{\kappa} u$ the wave front set in Gevrey class of order $\kappa$ of $u \in \mathscr{D}^{\prime}$ defined by Hörmander [1] (see Definition 1.1 in $\S 1$ ). In the present paper we shall show that the wave front set in Gevrey class of order smaller than 3 of a certain solution $U$ of the Cauchy problem (C.P)

Received October 8, 1984. 
propagates precisely along the curve $\tilde{C}_{0}$. (See Corollary 2.4 in $\S 2$ ). We remark that

$$
\tilde{C}_{0} \subset \Sigma_{1} \equiv\left\{\tilde{\rho} \in T^{*}\left(R_{t} \times R_{x}^{4}\right) \backslash 0 ; p_{1}(\tilde{\rho})=p_{2}(\tilde{\rho})=0\right\}
$$

and any Hamilton flow by $H_{p_{j}}(j=1,2)$ passing $\tilde{C}_{0}$ intersects $\Sigma_{1}$ only once with tangency of fourth order. Namely, $\tilde{C}_{0}$ is the generalized broken null-bicharacteristic flow (but not broken null-bicharacteristic flow).

The motivation of this study is derived from the work of Kumanogo-Taniguchi [7], where they constructed the fundamental solution of the Cauchy problem for a hyperbolic system of first order with diagonal principal part by using Fourier integral operators with multi-phases. Their fundamental solution gave the following description about the propagation of $C^{\infty}$-wave front sets of solutions; if we also denote by $U$ and $G$ the solution and the initial value of the Cauchy problem, respectively, then we have

$$
W F U\left(t_{0}\right) \subset \Gamma\left(t_{0} ; W F G\right), \quad t_{0}>0, \quad \text { (see Theorem } 3.4 \text { of [7]) . }
$$

Here $\Gamma\left(t_{0} ; \tilde{K}\right)$ for a closed conic set $\tilde{K}$ of $T^{*} R_{x}^{n} \mid 0$ is defined as follows: For $\varepsilon>0$ and integer $k \geqq 0$ we denote by $\Gamma_{\varepsilon}^{k}\left(t_{0} ; \tilde{K}\right)$ the set composed of end points (at $t=t_{0}$ ) of all $\varepsilon$-admissible trajectories of step $k$ issuing from $\tilde{K}$. We put

$$
\Gamma\left(t_{0} ; \tilde{K}\right)=\bigcap_{0<\varepsilon<1} \bigcup_{k=0}^{\infty} \Gamma_{\varepsilon}^{k}\left(t_{0} ; \tilde{K}_{\varepsilon}\right),
$$

where $\tilde{K}_{\varepsilon}$ denotes the $\varepsilon$-conic neighborhood of $\tilde{K}$ in $T^{*}\left(R_{x}^{n}\right) \backslash 0$. We refer the reader to [6] about the definition of $\varepsilon$-admissible trajectory for a hyperbolic system.

The description (0.5) seems to be rough to appearance because the set $\Gamma\left(t_{0} ; \tilde{K}\right)$ for a closed conic set $\tilde{K}$ contains the end point (at $t=t_{0}$ ) of a limiting curve $(\varepsilon \rightarrow 0)$ of the set composed of all $\varepsilon$-admissible trajectories issuing from $\tilde{K}_{\varepsilon}$. It should be noted, for hyperbolic system with characteristics of variable multiplicity, that the end point of the limiting curve is not always contained in the closure of the set of end points of all 0 -admissible trajectories issuing from $\tilde{K}$, that is,

$$
\left\{\begin{array}{l}
\text { there exist a } \delta_{\infty} \in T^{*} R_{x}^{n} \backslash 0 \text { and a closed conic set } \tilde{K} \\
\text { of } T^{*} R_{x}^{n} \backslash 0 \text { such that } \\
\delta_{\infty} \in \Gamma\left(t_{0} ; \tilde{K}\right) \backslash \overline{\bigcup_{k=0}^{\infty} \Gamma_{0}^{k}\left(t_{0} ; \tilde{K}\right)} .
\end{array}\right.
$$


In fact, examples satisfying the property (0.7) were given in [4] and [5]. (See last sections of [4] and [5]).

Let's return to the present example. If $\pi$ denotes the natural projection from $T^{*}\left(R_{t} \times R_{x}^{4}\right)$ to $R_{t} \times T^{*} R_{x}^{4}$ then the curve $\pi \tilde{C}_{0}$ is obtained as a limiting curve $(\varepsilon \rightarrow 0)$ of admissible trajectories issuing from $\varepsilon$-conic neighborhood of $\rho_{0}$ (see Propositions 2.1 and 2.2 in $\S 2$ ). If $\delta_{\infty}$ is the end point of $\pi \tilde{C}_{0}$ at $t=t_{0}$ and if $\tilde{K}$ is the right hand side of (0.3) then we have the property (0.7) and furthermore for any $1<\kappa<3$ there exists an initial value $G$ satisfying (0.3) such that

$$
W F_{\kappa} U\left(t_{0}\right) \ni \delta_{\infty},
$$

where $U$ is the solution of (C.P) (see Theorem 2.3 in $\S 2$ ). This fact shows that the description (0.5) is optimal for the present example if we consider the propagation of wave front sets in Gevrey class of order $1<\kappa<3$ instead of that of $C^{\infty}$ wave front sets. (See also Corollary 2.5 in $\S 2)$.

Recently, Wakabayashi [11] has given a description about the propagation of $C^{\infty}$ wave front sets of solutions of the Cauchy problem for symmetric hyperbolic systems, by using the work of Ivriǐ [3]. For hyperbolic systems of first order with diagonal principal part, the description of [11] is equivalent to (0.5). We remark that one direction of equivalence was proved in [11] (Theorem 4.4 of [11]) and another direction will be proved in the forthcoming paper [8] by K. Taniguchi and the author. The description of Wakabayashi is convenient to determine the set $\Gamma\left(t_{0} ; \cdot\right)$ for the present example (see $\S 6$ ). Finally we remark that the description (0.5) is also valid for wave front sets in Gevrey class if the symbol of hyperbolic system belongs to the corresponding Gevrey class. (See [8] and Wakabayashi [10]).

\section{$\S 1$. Wave front set in Gevrey class}

Let $\Omega$ be an open set in $R^{n}$ and let $\kappa \geqq 1$. We denote by $\gamma^{(x)}(\Omega)$ the set of all functions in Gevrey class of order $\kappa$, i.e. $u \in C^{\infty}(\Omega)$ and for any compact $K$ of $\Omega$ there exists a constant $C$ such that for any multi-index $\alpha$

$$
\sup _{x \in K}\left|D^{\alpha} u(x)\right| \leqq C^{|\alpha|+1}(\alpha !)^{x}
$$

According to the Definition 3.1 of Hörmander [1] we define the wave front set in Gevrey class of order $\kappa \geqq 1$. 
Definition 1.1. Let $\left(x^{0}, \xi^{0}\right) \in T^{*} R^{n} \backslash 0$ and let $u \in \mathscr{D}^{\prime}$. We say that $\left(x^{0}, \xi^{0}\right)$ is in the complement of the wave front set in Gevrey class of order $\kappa(\geqq 1)$ of $u$ if and only if there exist an open neighborhood $U_{0}$ of $x^{0}$, an open conic neighborhood $\Gamma_{0}$ of $\xi^{0}$ and a bounded sequence $\left\{u_{N}\right\} \subset \mathscr{E}^{\prime}$ such that

$$
\begin{cases}u_{N}=u & \text { in } U_{0} \\ \left|\hat{u}_{N}(\xi)\right| \leqq C^{N+1}(N !)^{x}|\xi|^{-N}, & \xi \in \Gamma_{0} \\ & N=1,2, \cdots\end{cases}
$$

for a constant $C>0$ independent of $N$. We denote by $W F_{k} u$ the wave front set in Gevrey class of order $\kappa$ of $u$.

Remark. When $\kappa=1$ the definition above is that of analytic wave front set.

In the same way as in the proof of Corollary 1.4 of Chapter V of Treves [9] we get the following lemma in view of (2.3) of [1].

Lemma 1.2. The point $\left(x^{0}, \xi^{0}\right) \in T^{*} R^{n} \backslash 0$ does not belong to the wave front set in Gevrey class of order $\kappa$ of $u \in \mathscr{D}^{\prime}$ if and only if there exist an open neighborhood $U_{0}$ of $x^{0}$ and an open conic neighborhood $\Gamma_{0}$ of $\xi^{0}$ such that we have the following property: For any sequence of functions $\left\{h_{N}\right\}$ $(N=1,2, \cdots)$ in $C_{0}^{\infty}\left(U_{0}\right)$ such that

$$
\left|D^{\alpha} h_{N}\right| \leqq\left(C_{1} N\right)^{x|\alpha|}, \quad|\alpha| \leqq N
$$

for a constant $C_{1}$ independent of $N$, we get

$$
\left|\left(\widehat{h_{N} u}\right)(\xi)\right| \leqq C_{2}^{N+1}(N !)^{k}|\xi|^{-N}, \quad \xi \in \Gamma_{0}
$$

for a constant $C_{2}$ independent of $N$.

Therefore we get the following

Corollary 1.3. Suppose that $\kappa>1$. Let $\left(x^{0}, \xi^{0}\right) \in T^{*} R^{n} \backslash 0$ and let $u \in \mathscr{D}^{\prime}$. Assume that there exist an increasing sequence of constants $\left\{C_{N}\right\}, a$ sequence $\left\{\zeta_{N} ; \zeta_{N} \in R^{n} \backslash 0\right\}$ and a sequence of functions $\left\{h_{N}\right\}$ in $C^{\infty}$ satisfying (1.3) $(N=1,2, \cdots)$ such that

$$
\left\{\begin{array}{l}
C_{N} \rightarrow \infty \quad(N \rightarrow \infty) \\
\zeta_{N} /\left|\zeta_{N}\right| \rightarrow \xi^{0} \| \xi^{0}|, \quad| \zeta_{N} \mid \rightarrow \infty \quad(N \rightarrow \infty)
\end{array}\right.
$$




$$
\operatorname{supp} h_{N} \rightarrow\left\{x^{0}\right\} \quad(N \rightarrow \infty)
$$

and

$$
\left|\left(\widehat{h_{N} u}\right)\left(\zeta_{N}\right)\right| \geqq C_{N}^{N+1}(N !)^{\kappa}\left|\zeta_{N}\right|^{-N}, \quad N=1,2, \cdots .
$$

Then $\left(x^{0}, \xi^{0}\right)$ belongs to $W F_{\kappa} u$.

Remark. Let $\left\{N_{k}\right\}$ be a subsequence of $\{1,2,3, \cdots\}$. Clearly we have the same conclusion even if we replace $\{N\}$ by $\left\{N_{k}\right\}$ in the corollary.

\section{§2. Results}

Let $L$ be the hyperbolic system in $R_{t} \times R_{x}^{4}$ defined by (0.1) and (0.2). Let $t_{0}$ be any (but a fixed) positive. For an even integer $\mu=2,4,6, \cdots$, we put

$$
\rho_{\mu}=\left(0, \eta^{\mu}\right) \in T^{*} R_{x}^{4}, \quad \eta^{\mu}=\left(-\left(t_{0} / 2 \mu\right)^{4}, 0,0,1\right) .
$$

Proposition 2.1. As admissible trajectories issuing from $\rho_{\mu}$ one can find only the following $\mu+2$ trajectories; two bicharacteristic curves with respect to $\lambda_{1}$ and $\lambda_{2}$, issuing from $\rho_{\mu}$, and trajectories $C\left(J_{r}^{\prime}, \tilde{t}_{r}^{\prime}, \rho_{\mu}\right)$ of step $r=1, \cdots, \mu$. Here $J_{r}^{\prime}=(1,2, \cdots, 1)$ or $(2,1,2, \cdots, 1)$ according to $r$ even or odd, and $\tilde{t}_{r}^{\prime}=\left(t_{1}^{r}, \cdots, t_{r}^{r}\right)=\left(\sigma_{1}, \cdots, \sigma_{r}\right), \quad \sigma_{p}=(2 q-1) \beta \quad(q=1, \cdots, \mu$, $\left.\beta=\left(t_{0} / 2 \mu\right)\right)$. (See Fig. 1 and 2). Furthermore, for the trajectory $C_{\mu}=$ $C\left(J_{\mu}^{\prime}, \tilde{t}_{\mu}^{\prime}, \rho_{\mu}\right)$ all hypotheses of Theorem 1 of [6] are verified with $\rho_{0}=\rho_{\mu}$.

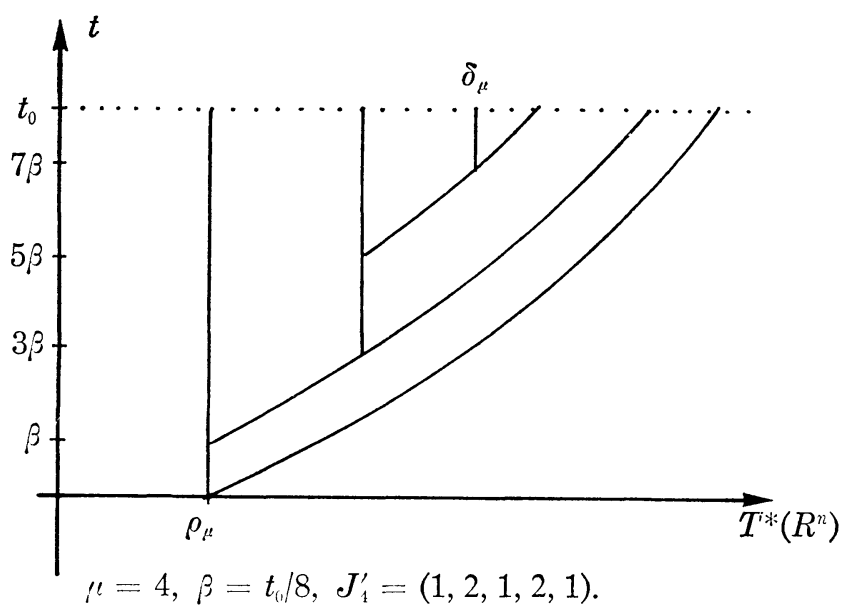

Fig. 1. 


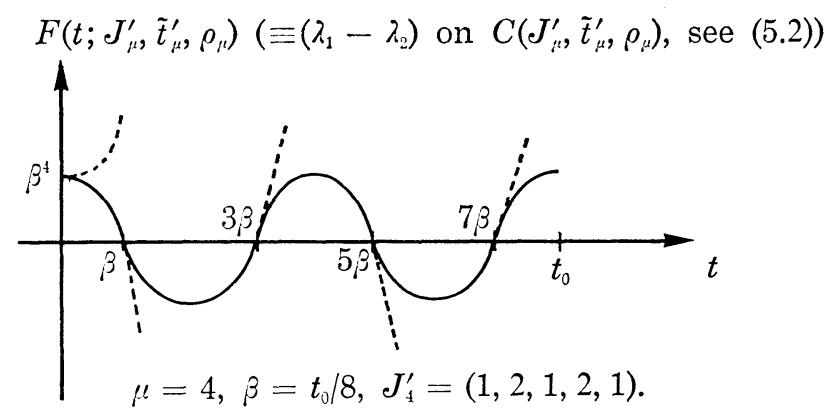

Fig. 2.

As the positive $\sigma$ and the neighborhood $V_{0}$ in the hypothesis $(\mathrm{H}-3)$ we can take an arbitrary positive smaller than 1 and $R_{x}^{4}$, respectively.

We denote by $\delta_{\mu}$ the end point of the trajectory $C\left(J_{\mu}^{\prime}, \tilde{t}_{u}^{\prime}, \rho_{\mu}\right)$ and consider a sequence of end points $\left\{\delta_{\mu}\right\}(\mu=2,4,6, \cdots)$. Let $\tilde{\rho}_{0} \in T^{*}\left(R_{t} \times R_{x}^{4}\right)$ be $(0,(0,0,0,0,1))$ and let $\tilde{C}_{0}$ be an integral curve through $\tilde{\rho}_{0}$ by the Hamilton field $H_{p_{1}+p_{2}}$.

Proposition 2.2. i) There exists a unique limiting point $\delta_{\infty}=\lim _{\mu \rightarrow \infty} \delta_{\mu}$. The limiting point $\delta_{\infty}$ equals the end point of the curve $\pi \tilde{C}_{0} \subset R_{t} \times T^{*} R_{x}^{4}$ at $t=t_{0}$, where $\pi$ is the natural projection from $T^{*}\left(R_{t} \times R_{x}^{4}\right)$ to $R_{t} \times T^{*} R_{x}^{4}$.

ii) It follows that

$$
\tilde{C}_{0} \subset \Sigma_{1} \equiv\left\{\tilde{\rho} \in T^{*}\left(R_{t} \times R_{x}^{4}\right) \backslash 0 ; p_{1}(\tilde{\rho})=p_{2}(\tilde{\rho})=0\right\}
$$

and any Hamilton flow of $H_{p_{j}}(j=1,2)$ passing $\tilde{C}_{0}$ intersects only once with osculation of fourth order.

iii) Let $\tilde{C}_{0}^{+}$be the part of the curve $\tilde{C}_{0}$ corresponding to $t \geqq 0$ and let $B_{\tilde{\rho}_{0}}$ be a bundle of half hamilton flows by $H_{p_{j}}(j=1,2)$ emanating from $\tilde{C}_{0}^{+}$, that is,

$$
B_{\tilde{\rho}_{0}}=\bigcup_{j=1,2}\left\{\left(\exp s H_{p_{j}}\right) \tilde{\rho} ; s \geqq 0, \tilde{\rho} \in \tilde{C}_{0}^{+}\right\} .
$$

If $\tilde{K}$ is a closed conic subset in $T^{*} R_{x}^{4} \backslash 0$

$$
\left\{c \rho_{0} \equiv\left(o, c \eta^{0}\right) ; c>0, \eta^{0}=(0,0,0,1)\right\}
$$

then it follows that

$$
\Gamma\left(t_{0} ; \tilde{K}\right)=\left\{(x, c \xi) \in T^{*} R_{x}^{4} \backslash 0 ; c>0,\left.(x, \xi) \in \pi B_{\tilde{\rho}_{0}}\right|_{t=t_{0}}\right\},
$$

where $\left.\pi B_{\tilde{\rho}_{0} \mid}\right|_{t=t_{0}}$ is the section of $\pi B_{\tilde{p}_{0}} \subset R_{t} \times T^{*} R_{x}^{4}$ at $t=t_{0}$. 
Let's admit these propositions, which will be proved in Section 5 and Section 6 , respectively.

THeOREm 2.3. If $\tilde{K}$ is the closed conic set defined by (2.4) and $\delta_{\infty}=$ $\lim _{\mu \rightarrow \infty} \delta_{\mu}$ then we get

$$
\delta_{\infty} \in \Gamma\left(t_{0} ; \tilde{K}\right) \backslash \overline{\bigcup_{k=0}^{\infty} \Gamma_{0}^{k}\left(t_{0} ; \tilde{K}\right)} .
$$

Furthermore, for any positive $1<\kappa_{0}<3$ there exists an initial value $G \in \mathscr{E}^{\prime}$ such that

$$
W F G=W F_{\kappa_{0}} G=\tilde{K}
$$

and

$$
\delta_{\infty} \in W F_{\kappa^{\prime}} U\left(t_{0}\right) \quad \text { for any } \kappa_{0} \leqq \kappa^{\prime}<3,
$$

where $U$ is the solution of the Cauchy problem (C.P).

Corollary 2.4. For any $1<\kappa_{0}<3$ there exists an initial value $G \in \mathscr{E}^{\prime}$ satisfying (2.7) such that

$$
\tilde{C}_{0} \subset W F_{\kappa^{\prime}} U \quad\left(\subset T^{*}\left(R_{t} \times R_{x}^{4}\right) \backslash 0\right) \quad \text { for } \kappa_{0} \leqq \kappa^{\prime}<3,
$$

where $U$ is the solution of (C.P).

Corollary 2.5. For any $1<\kappa_{0}<3$ there exists an initial value $G \in \mathscr{E}^{\prime}$ satisfying (2.7) such that

$$
W F_{\kappa^{\prime}} U\left(t_{0}\right)=\Gamma\left(t_{0} ; \tilde{K}\right) \quad \text { for any } \kappa_{0} \leqq \kappa^{\prime}<3,
$$

where $U$ is the solution of (C.P).

\section{§3. Plan of the proof of Theorem 2.3}

Put $\mu=2 N$ for $N=1,2,3, \cdots$ and $\eta_{N}=\eta^{\mu}$,

$$
\eta_{N}=\left(-\left(t_{0} / 4 N\right)^{4}, 0,0,1\right) \text {. }
$$

We put

$$
\phi(z)=(2 \pi)^{-2} \exp \left(-\sum_{j=1}^{4} z_{\jmath}^{2} / 2\right), \quad z \in C^{4}
$$

and

$$
g(z)=\sum_{N=1}^{\infty} \tau_{N}^{-1} \phi\left(\tau_{N}^{1-r} z\right) \exp i \tau_{N} z \cdot \eta_{N}
$$


where $\gamma$ is a positive satisfying

$$
0<\gamma<1 / 2
$$

and $\left\{\tau_{N}\right\}_{N=1}^{\infty}$ is an increasing sequence of positive numbers such that $\tau_{N+1} / \tau_{N}$ is large enough to satisfy conditions demanded later on. Let $g(x)$ be the restriction of $g(z)$ to $R^{4}$. The function $g(x)$ is also a modification of Example 2.3 of Hörmander [2]. In the similar manner as in [6] we get

$$
W F g=W F_{\kappa_{0}} g=\left\{\left(0, c \eta^{0}\right) ; c>0, \eta^{0}=(0,0,0,1)\right\},
$$

where $\kappa_{0}=1 / 2 \gamma>1$. Indeed, note that $\phi(z) \exp i z \cdot \eta$ for $|\eta| \leqq 2$ is bounded in an open set of $C^{4}$

$$
\Omega_{0}=\left\{z \in C^{4} ;|\operatorname{Re} z|^{2}>|\operatorname{Im} z|^{2}+4|\operatorname{Im} z|\right\} .
$$

Since we may assume $\sum \tau_{N}^{-1}<\infty$ the sum of the right hand side of (3.2) converges uniformly in $\Omega_{0}$. Therefore $g(z)$ is analytic in $\Omega_{0}$ and $g(x)$ is real analytic except the origin. Now we have

$$
\hat{g}(\xi)=\sum_{N} \tau_{N}^{4(\gamma-1)-1} \hat{\phi}\left(\left(\xi-\tau_{N} \eta_{N}\right) \tau_{N}^{\gamma-1}\right) .
$$

Since we have

$$
\hat{\phi}(\xi)=\exp \left(-|\xi|^{2} / 2\right) \geqq 0
$$

it follows that

$$
\hat{g}\left(\tau_{N} \eta_{N}\right) \geqq \tau_{N}^{4(\gamma-1)-1}
$$

which shows

$$
\left\{\left(0, c \eta^{0}\right) ; c>0\right\} \subset W F g \subset W F_{\kappa_{0}} g .
$$

Let $V$ be an open conic neighborhood of $\eta^{0}$. By the same way as in (2.17) of [6] we have for a positive constant $C_{V}$

$$
\left|\xi-\tau_{N} \eta_{N}\right| \tau_{N}^{\gamma-1} \geqq C_{V}|\xi|^{r}
$$

if $\xi \notin V$ and $N$ is large enough. It follows from (3.5) that there exists a constant $c_{0}>0$ such that

$$
|\hat{g}(\xi)| \leqq c_{0}^{-1} \exp \left(-c_{0}|\xi|^{2 \gamma}\right) \quad \text { if } \xi \notin V,
$$

which shows another direction of inclusion of (3.4).

We choose, as the initial value $G$ of (C.P), 


$$
G(x)={ }^{t}\left(\psi_{0}(x) g(x), \psi_{0}(x) g(x)\right),
$$

where $\psi_{0}(x)$ is a function in $\gamma^{\left(\kappa_{0}\right)} \cap C_{0}^{\infty}\left(\kappa_{0}=1 / 2 \gamma\right)$ such that $0 \leqq \psi_{0} \leqq 1$, $\psi_{0}(x)=1$ for $|x| \leqq R_{0}$. Here $R_{0}$ is a positive large number. Clearly we get $G \in \mathscr{E}^{\prime}$ and

$$
W F_{\kappa_{0}} G=W F G=\left\{\left(0, c \eta^{0}\right) ; c>0, \eta^{0}=(0,0,0,1)\right\} .
$$

We put $\delta_{\mu}=\delta_{2 N}=\left(x_{N}, \xi_{N}\right)$ and $\delta_{\infty}=\left(x^{0}, \xi^{0}\right)$. Note that $\delta_{\infty}=\lim _{N \rightarrow \infty} \delta_{2 N}$ by means of Proposition 2.2. Let $\left\{f_{N}(x)\right\}(N=1,2, \cdots)$ be a sequence of functions in $C_{0}^{\infty}$ such that $0 \leqq f_{N} \leqq 1, f_{N}=1$ on $\left|x-x^{0}\right| \leqq 1, f_{N}=0$ except $\left|x-x^{0}\right| \leqq 2$ and for any $\alpha$

$$
\left|D^{\alpha} f_{N}\right| \leqq(C N)^{|\alpha|}, \quad|\alpha| \leqq N
$$

for a constant $C>0$ independent of $N$. It is easy to construct $\left\{f_{N}\right\}$ (see Lemma 1.1 of Chapter $\mathrm{V}$ of [9]). Let $\left\{\beta_{N}\right\}_{N=1}^{\infty}$ be an increasing sequence of positive numbers such that $\beta_{N} \leqq \log N$. If we put

$$
h_{N}(x)=f_{N}\left(x^{0}+\beta_{N}\left(x-x^{0}\right)\right),
$$

the sequence $\left\{h_{N}\right\}$ satisfies conditions (1.3) and (1.6). We may assume that $\beta_{N}$ is slowly increasing such that

$$
h_{N}=1 \quad \text { near } x_{N} .
$$

Let $U={ }^{t}\left(u_{1}, u_{2}\right)$ be the solution of (C.P) for the $G$ defined by (3.8) and (3.2). We shall show that for any $N$ there exists a constant $c_{1}>0$ independent of $N$ such that

$$
\left|\left(\widehat{h_{N} u_{1}}\right)\left(t_{0}, \zeta_{N}\right)\right| \geqq c_{1}^{N+1} N^{3 N}\left|\zeta_{N}\right|^{-N+4(\gamma-1)-1},
$$

where $\zeta_{N}=\tau_{N} \xi_{N}$. Admitting this, by means of Corollary 1.3 we can obtain the last part of Theorem 2.3. The first part of Theorem 2.3 will be proved in Section 5 with Proposition 2.1.

\section{§4. Proof of (3.12)}

When we consider $h_{N} u_{1}\left(t_{0}, x\right)$, in view of (2.4) of [6] we may assume $G={ }^{t}(g, g)$ instead of (3.8). Indeed, we have

$$
h_{N}(x)\left(1-\psi_{0}\left(\pi_{J_{\nu}}\left(\tilde{t}_{\nu}\right) x\right)\right)=0
$$

if $R_{0}$ is large enough. By the same way as in getting (2.20) of [6] we have 


$$
\begin{aligned}
\left(\widehat{h_{N} u_{1}}\right)\left(t_{0} ; \tau_{N} \xi_{N}\right)= & \sum_{N^{\prime}} \tau_{N^{\prime}}^{4}(\gamma-1)-1\left(w_{(1)}\left(\tau_{N}, \tau_{N^{\prime}}\right)\right. \\
& \left.+\sum_{\nu=1}^{\infty} \sum_{\substack{\nu \in \Pi_{\nu}^{0} \\
j_{\nu}=1}} \int_{\Delta_{\nu}} w_{J_{\nu}}\left(\tau_{N}, \tau_{N^{\prime}} ; \tilde{t}_{\nu}\right) d \tilde{t}_{\nu}\right),
\end{aligned}
$$

where $w_{J_{\nu}}\left(\tau_{N}, \tau_{N^{\prime}} ; \tilde{t}_{\nu}\right)=G_{J_{\nu}}\left(\tau_{N}, \tau_{N^{\prime}} ; \tilde{t}_{\nu}\right) \exp i \tau_{N} F_{J_{\nu}}\left(\tilde{t}_{\nu}\right)\left(\nu \geqq 0, J_{0}=(1)\right)$,

$$
F_{J_{\nu}}\left(\tilde{t}_{\nu}\right)=-\xi_{N} \cdot d_{J_{\nu}}\left(\tilde{t}_{\nu}\right) \quad\left(\text { note } y^{0}=0\right)
$$

and

$$
\begin{aligned}
G_{J_{\nu}}\left(\tau_{N}, \tau_{N^{\prime}} ; \tilde{t}_{\nu}\right)= & \int \exp \left(i y \cdot\left(\tau_{N^{\prime}} \eta_{N^{\prime}}-\tau_{N}{ }^{t} M_{J_{\nu}}\left(\tilde{t}_{\nu}\right) \xi_{N}\right) \tau_{N^{\prime}}^{\gamma-1}\right) \\
& \times h_{N}\left(M_{J_{\nu}}\left(\tilde{t}_{\nu}\right)\left(\tau_{N^{\prime}}^{\gamma-1} y\right)+d_{J_{\nu}}\left(\tilde{t}_{\nu}\right)\right) \phi(y)(-i)^{\nu} d y .
\end{aligned}
$$

Here we used $\beta_{J_{\nu}^{\prime}}=(-i)^{\nu}$ because $\operatorname{det} M_{J_{\nu}}=1$. By the similar way as in (2.24) of [6] we get for a $\gamma_{0}$ satisfying $0<\gamma_{0}<\gamma$

$$
\left|\tau_{N^{\prime}} \eta_{N^{\prime}}-\tau_{N}{ }^{t} M_{J_{\nu}}\left(\tilde{t}_{\nu}\right) \xi_{N}\right| \tau_{N^{\prime}}^{\gamma-1} \geqq \tau_{N}^{\gamma_{0}}
$$

if $\left|\tau_{N}-\tau_{N^{\prime}}\right|$ is large enough. We remark that directions of $\eta_{N^{\prime}}$ and $\xi_{N}$ do not concern the derivation of (4.4). Integrating by parts with respect to $y\left[(N+3+4(\gamma-1)) / \gamma_{0}\right]$ times, we obtain

$$
\begin{aligned}
\left|G_{J_{\nu}}\left(\tau_{N}, \tau_{N^{\prime}} ; \tilde{t}_{\nu}\right)\right| \leqq & C_{N}^{\nu} \tau_{N}^{-(N+2)+4(\gamma-1)} \\
& \text { if }\left|\tau_{N}-\tau_{N^{\prime}}\right| \text { large enough, }
\end{aligned}
$$

where $C_{N}$ is a constant independent of $\nu$ and $N^{\prime}$. Note that $\sum \tau_{N^{\prime}}^{4(\gamma-1)-1}<$ $+\infty$ and that the volume of $\Delta_{\nu}$ is $t_{0}^{\nu} / \nu$ !. If we denote by $R\left(\tau_{N}\right)$ the sum with respect to $N^{\prime} \neq N$ in the right hand side of (4.1), there exists a constant $C_{N}^{\prime}$ such that

$$
\left|R\left(\tau_{N}\right)\right| \leqq C_{N}^{\prime} \tau_{N}^{-(N+2)+4(\gamma-1)}
$$

if $\left|\tau_{N}-\tau_{N^{\prime}}\right|$ for $N^{\prime} \neq N$ is so large that the inequality (4.4) holds.

From now on we shall consider the term with $N^{\prime}=N$ in the right hand side of (4.1), that is,

$$
\tau_{N}^{4(\gamma-1)-1} \sum_{\nu=0}^{\infty} I_{J_{\nu}}\left(\tau_{N}\right), \quad\left(J_{0}=(1), J_{\nu} \in \Pi_{\nu}^{0} \text { such that } j_{1}=1\right),
$$

where $I_{J_{\nu}}(\tau)$ is defined by (2.26) of [6] with $G_{J_{\nu}}$ as follows:

$$
\begin{aligned}
G_{J_{\nu}}\left(\tau ; \tilde{t}_{\nu}\right)= & (-i)^{\nu} \int \exp i \tau^{\gamma}\left(\eta_{N}-{ }^{t} M_{J_{\nu}}\left(\tilde{t}_{\nu}\right) \xi_{N}\right) \cdot y \\
& \times h_{N}\left(M_{J_{\nu}}\left(\tilde{t}_{\nu}\right)\left(\tau^{\gamma-1} y\right)+d_{J_{\nu}}\left(\tilde{t}_{\nu}\right)\right) \phi(y) d y .
\end{aligned}
$$


Since it follows from Proposition 2.1 that, for the trajectory $C\left(J_{\mu}^{\prime}, \tilde{t}_{\mu}^{\prime}, \rho_{\mu}\right)$ $(\mu=2 N)$, all hypotheses of Theorem 1 of [6] are satisfied, we can use the same method as in Section 3 of [6] and obtain the result corresponding to Lemma 2.1 of [6], that is; there exist two constant $c_{\mu}>0$ and $C_{\mu}>0$ independent of $\nu$ such that

$$
\left|I_{J^{\prime} \mu}(\tau)\right| \geqq c_{\mu} \tau^{-\mu / 2} \quad \text { if } \tau \geqq \tau(\mu)
$$

and for any $J_{\nu} \neq J_{\mu}^{\prime}$

$$
\left|I_{J_{\nu}}(\tau)\right| \leqq C_{\mu}^{\nu} \tau^{-\mu / 2-\sigma_{\gamma} / 4} / \nu ! \quad \text { if } \tau \geqq \tau(\mu),
$$

where $\tau(\mu)$ is a large number depending on $\mu=2 N$. Here we used (3.11) to show $c_{\mu}>0$ (see Remark 1 in $\S 3$ of [6]).

Since $\mu=2 N$, the conjunction of (4.6), (4.8) and (4.9) shows that there exists a constant $C_{N}^{\prime \prime}>0$ such that

$$
\left|\left(\widehat{h_{N}} u_{1}\right)\left(t_{0} ; \tau_{N} \xi_{N}\right)\right| \geqq\left(c_{2 N}-C_{N}^{\prime \prime} \tau_{N}^{-\sigma} \sigma^{/ 4}\right) \tau_{N}^{-N+4(\gamma-1)-1}
$$

if $\left\{\tau_{N}\right\}$ satisfies

$$
\left\{\begin{array}{l}
\tau_{N} \geqq \tau(2 N) \\
\left|\tau_{N}-\tau_{N^{\prime}}\right|\left(N \neq N^{\prime}\right) \quad \text { large enough to get (4.4). }
\end{array}\right.
$$

If we choose $\left\{\tau_{N}\right\}$ furthermore such that

$$
c_{2 N}-C_{N}^{\prime \prime} \tau_{N}^{-\sigma \gamma / 4} \geqq c_{2 N} / 2,
$$

for the proof of (3.12) it remains to show that for any $N$ there exists a constant $c_{1}>0$ independent of $N$ such that

$$
c_{2 N}=c_{1}^{N} N^{3 N} \quad\left(\text { or } c_{\mu}=c_{1}^{\mu / 2}(\mu / 2)^{3 \mu / 2}\right) .
$$

In consideration of the formula (3.35) of [6] and Remark 1 of Section 1 of [6], it suffices to show that for any $\mu=2 N$ there exists a constant $c_{2}>0$ independent of $\mu$ such that

$$
c_{2}^{-\mu} \mu^{-3 \mu} \leqq\left|\operatorname{det}\left(\partial_{t_{p}} \partial_{t q} \xi_{N} \cdot d_{J_{\mu}^{\prime \mu}}\left(\tilde{t}_{\mu}^{\prime}\right)\right)\right| \leqq c_{2}^{\mu} \mu^{-3 \mu} .
$$

This inequality will be proved in the next section.

\section{§5. Proof of Proposition 2.1}

If $H_{j}$ denotes the Hamilton vector field of $p_{j}$, that is, $H_{j}=H_{p_{j}}$ $(j=1,2)$ then it follows that 


$$
H_{j_{1}} H_{j_{2}} \cdots H_{j_{5}} p_{j_{6}}=0, \quad j_{k} \in\{1,2\} .
$$

The condition (N.m) with integer $m \geqq 1$ was already defined in Introduction of [4], to which we refer the reader about motivation of the definition and results under the condition with $m \leqq 3$. By the same way as in the proof of Lemma 2.1 of [4] we get

Lemma 5.1. If the condition (N.5) is verified then $H_{j_{1}} H_{j_{2}} \cdots H_{j_{4}} p_{j_{5}}$ $\left(j_{k} \in\{1,2\}\right)$ are constants along any trajectory.

By this lemma we see that along any trajectory issuing from $\rho_{\mu}$

$$
\left\{\begin{array}{l}
H_{1}^{4} p_{2}=H_{2}^{4} p_{1}=H_{1}^{2} H_{2}^{2} p_{1}=H_{2}^{2} H_{1}^{2} p_{2}=24 \\
H_{2} H_{1}^{3} p_{2}=H_{1} H_{2}^{3} p_{1}=H_{1} H_{2} H_{1}^{2} p_{2}=H_{2} H_{1} H_{2}^{2} p_{1}=-24 .
\end{array}\right.
$$

Let $\left\{(t, x(t), \xi(t)) ; t \in\left[0, t_{0}\right]\right\} \subset R_{t} \times T^{*} R_{x}^{4}$ be a trajectory $C\left(J_{\nu}, \tilde{t}_{\nu}, \rho_{\mu}\right)$. Then $H_{1}^{3} p_{2}(t, x(t), \xi(t)),-H_{2}^{3} p_{1}(t, x(t), \xi(t))$ and $H_{1} H_{2}^{2} p_{1}(t, x(t), \xi(t))\left(\equiv-H_{2} H_{1}^{2} p_{2}(t, x(t)\right.$,

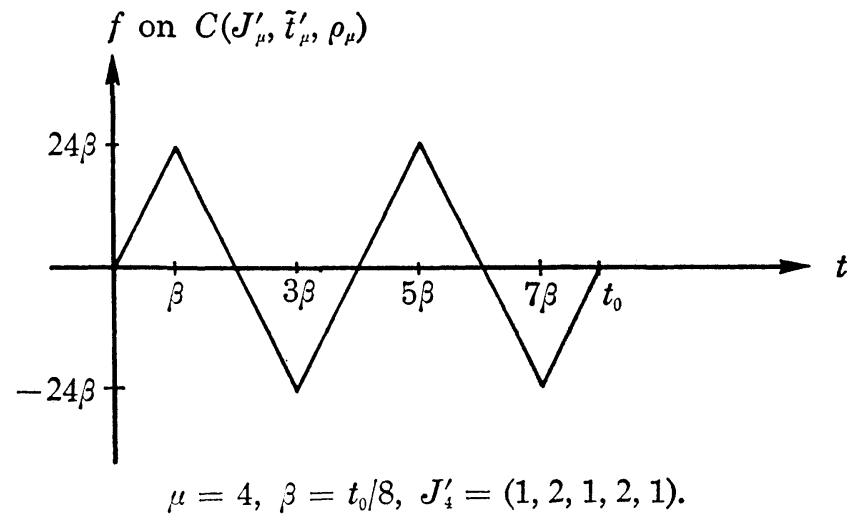

Fig. 3.

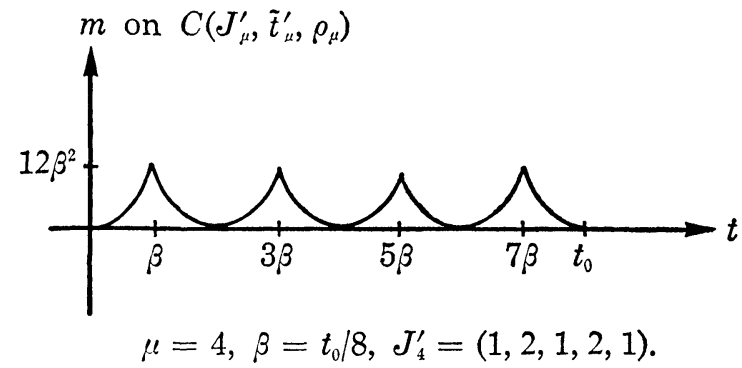

Fig. 4. 
$\xi(t)$ ), which follows from the Jacobi identity) are equal to $f(t)$, a continuous piecewise linear function such that $f^{\prime}(t)$ on $\left(t_{q}, t_{q-1}\right)$ is 24 or -24 according to $j_{q}=1$ or 2 and $f(0)=0$. Indeed, by means of (5.1) we can see, for example, that $d\left(H_{1}^{3} p_{2}\right) / d t$ on $\left(t_{q}, t_{q-1}\right)$ is equal to $H_{1}^{4} p_{2}=24$ or $H_{2} H_{1}^{3} p_{2}$ $=-24$ according to $j_{q}=1$ or 2 . Clearly $H_{1}^{3} p_{2}\left(0, \rho_{\mu}\right)=0$ and it follows from (N.5) that $d^{2}\left(H_{1}^{3} p_{2}\right) / d t^{2}=0$. (See Fig. 3).

On the other hand, it follows from (N.5) and (5.1) that $H_{1}^{2} p_{2}(t, x(t), \xi(t))$ and $H_{2}^{2} p_{1}(t, x(t), \xi(t))$ are equal to $m(t)$ a continuous piecewise quadratic function such that $m(0)=0, m^{\prime \prime}=24$ and $m^{\prime}$ on $\left(t_{q}, t_{q-1}\right)$ is equal to $f$ or $-f$ according to $j_{q}=1$ or 2. (See Fig. 4)

If we put

$$
F\left(t ; J_{\nu}, \tilde{t}_{\nu} \rho_{\mu}\right)=\left(\lambda_{1}-\lambda_{2}\right)(t, x(t), \xi(t))
$$

for the trajectory $C\left(J_{\nu}, \tilde{t}_{\nu}, \rho_{\mu}\right)=\left\{(t, x(t), \xi(t)) ; t \in\left[0, t_{0}\right]\right\}$ then $F$ is a continuously differentiable function of degree 1 and piecewise polynomials of order 4 such that

$$
\left\{\begin{array}{l}
F^{\prime \prime \prime \prime}=24 \text { or }-24, \quad F^{\prime \prime \prime}=-f \text { or } f \\
F^{\prime \prime}=-m \text { or } m \text { on }\left(t_{q}, t_{q-1}\right) \text { according to } j_{q}=1 \text { or } 2,
\end{array}\right.
$$

because $d^{j} F / d t^{j}(j=1,2, \cdots)$ are equal to $H_{1}^{j}\left(p_{1}-p_{2}\right)=-H_{1}^{j} p_{2}$ or $H_{2}^{j}\left(p_{1}-p_{2}\right)=H_{2}^{j} p_{1}$ on $\left(t_{q}, t_{q-1}\right)$ according to $j_{q}=1$ or 2 and because we have $H_{1} p_{2}=-H_{2} p_{1}$ trivially.

Noting that $H_{1} p_{2}\left(0, \rho_{\mu}\right)=0$ and $\left(\lambda_{1}-\lambda_{2}\right)\left(0, \rho_{\mu}\right)=\beta^{4}\left(\beta=t_{0} / 2 \mu\right)$ we get

$$
\left\{\begin{array}{l}
F_{-}(t) \equiv F\left(t ;(1), \emptyset, \rho_{\mu}\right)=\beta^{4}-t^{4} \\
F_{+}(t) \equiv F\left(t ;(2), \emptyset, \rho_{\mu}\right)=\beta^{4}+t^{4} \quad \text { (see Fig. 2), }
\end{array}\right.
$$

where we denote by $C\left((1), \emptyset, \rho_{\mu}\right)$ and $C\left((2), \emptyset, \rho_{\mu}\right)$ two bicharacteristic curves with respect to $\lambda_{1}$ and $\lambda_{2}$, respectively. Furthermore, for $r=1, \cdots, \mu$, we have

$$
\begin{aligned}
F_{r}(t) \equiv F\left(t ; J_{r}^{\prime}, \tilde{t}_{r}^{\prime}, \rho_{\mu}\right)=(-1)^{r-q}\left(\beta^{4}-(t-2(r-q) \beta)^{4}\right) \\
\quad \text { on } t \in\left[t_{q}^{r}, t_{q-1}^{r}\right]\left(q=1, \cdots, r+1, t_{r+1}^{r}=0, t_{0}^{r}=t_{0}\right) .
\end{aligned}
$$

(See Fig. 2) .

By means of (5.3)-(5.5) we get the first part of Proposition 2.1. The hypothesis (H-1) of Theorem 1 of [6] follows from (5.5). By the similar way as in getting Proposition 5.2 of [4], in view of (5.3)-(5.5) we get (5.3) of [4], which shows that the hypothesis (H-3) of Theorem 1 of [6] is satisfied. 
As the $\sigma$ of (H-3) we can take an arbitrary positive smaller than 1 because constant times $\varepsilon$ is inferior to $\varepsilon^{\sigma}$ for small $\varepsilon<1$. As the $V_{0}$ of (H-3) we can take $R_{x}^{4}$ because the $\xi$ variables of end points of admissible trajectories issuing from $\rho_{\mu}$ are different each other, which is easily checked by the direct calculation if we take notice of the $\xi_{3}$ component.

Before the check of the hypothesis (H-2) of Theorem 1 we shall show the first part of Theorem 2.3. By the same way as in the last paragraph of Section 7 of [4], noting that $\left(\lambda_{1}-\lambda_{2}\right)\left(0, \rho_{0}\right)=0\left(\rho_{0}=\left(0, \eta^{0}\right)\right)$ we have by (5.3) and (5.4)

$$
\overline{\bigcup_{k} \Gamma_{0}^{k}\left(t_{0} ; \tilde{K}\right)}=\overline{\Gamma_{0}^{0}\left(t_{0} ; \tilde{K}\right)}=\Gamma_{0}^{0}\left(t_{0} ; \tilde{K}\right)
$$

for the set $\tilde{K}$ defined by (2.4). We get

$$
\delta_{\infty} \notin \Gamma_{0}^{0}\left(t_{0} ; \tilde{K}\right) \text {, }
$$

because $\lambda_{1}-\lambda_{2}=0$ at $\left(t_{0}, \delta_{\infty}\right)$ and it is not zero on $\Gamma_{0}^{0}\left(t_{0} ; \tilde{K}\right)$.

Now we shall check the hypothesis $(\mathrm{H}-2)$, that is,

$$
c_{2}^{-\mu} \mu^{-3 \mu} \leqq\left|\operatorname{det}\left(\partial_{t_{p}} \partial_{t_{q}} \xi^{\mu} \cdot d_{J_{\mu}^{\prime}}\left(\tilde{t}_{\mu}^{\prime}\right)\right)\right| \leqq c_{2}^{\mu} \mu^{-3 \mu}
$$

for a constant $c_{2}>0$ independent of $\mu$. Here we putted $\delta_{\mu}=\left(x^{\mu}, \xi^{\mu}\right)$ $\left(=\delta_{2 N}=\left(x_{N}, \xi_{N}\right)\right)$. We recall that $F_{J^{\prime}}\left(\tilde{t}_{\mu}^{\prime}\right)=-\xi^{\mu} \cdot d_{J_{\mu}^{\prime}}\left(\tilde{t}_{\mu}\right)$. By means of (3.13) and (3.14) of [6] with $y^{0}=0, \eta^{0}=\eta^{\mu}$ and $\xi^{0}=\xi^{\mu}$ we get

$$
\partial_{t_{q}} F_{J_{\mu}^{\prime}}=\left(\lambda_{j_{q}}-\lambda_{j_{q+1}}\right)\left(t_{q}, x^{q}, \xi^{q}\right)+\left({ }^{t} M_{J_{\mu}^{\prime}}^{-1} \eta^{\mu}-\xi^{\mu}\right) \cdot \partial_{t_{q}} d_{J_{\mu}^{\prime}} \cdot
$$

Differentiating with respect to $t_{p}$ and putting $\tilde{t}_{\mu}=\tilde{t}_{\mu}^{\prime}$ we have

$$
\begin{aligned}
a_{p, q} & \equiv \partial_{t_{p}} \partial_{t_{q}} F_{J_{\mu}^{\prime}}\left(\tilde{t}_{\mu}^{\prime}\right) \\
& =\left(t_{0}^{3} / 2 \mu^{3}\right) \delta_{p, q}+\left(\partial_{t_{p}}\left({ }^{t} M_{J_{\mu}^{\prime}}^{-1} \eta^{\mu}\right) \cdot \partial_{t_{q}} d_{J_{\mu}^{\prime}}\right)\left(\tilde{t}_{\mu}^{\prime}\right) \quad \text { if } p \leqq q,
\end{aligned}
$$

where we used $\left.\left(d F\left(t ; J_{\mu}^{\prime}, t_{\mu}^{\prime}, \rho_{\mu}\right) / d t\right)\right|_{t=(2 \mu-2 q+1) \beta}=(-1)^{q-1} 4 \beta^{3}$ to obtain the derivative of the first term of the right hand side of (5.8). Here $\delta_{p, q}$ is the $\delta$ of Kronecker. Since the direct calculation shows that the second term of the right hand side of (5.9) is equal to $(-1)^{q-1}$ multiplied by a constant independent of $q$, we get by (5.9)

$$
a_{p, q}+a_{p, q+1}=\left(t_{0} / 2 \mu^{3}\right) \delta_{p, q} \quad \text { if } p \leqq q .
$$

Furthermore, the direct calculation gives

$$
a_{1,1}=(4 \mu-1) t_{0}^{3} / 2 \mu^{3}, \quad a_{p-1, p}+a_{p, p}=-3 t_{0}^{3} / 2 \mu^{3}, \quad 2 \leqq p \leqq \mu .
$$


If we denote by $T_{\mu}$ a $\mu \times \mu$ matrix

$$
\left(\begin{array}{rrrrrr}
1 & 0 & & & 0 \\
1 & 1 & & & 0 \\
& 1 & \ddots & & \\
& & \ddots & & \\
0 & & \ddots & 0 \\
& & & 1 & 1
\end{array}\right)
$$

then it follows from (5.10), (5.11) and $a_{p, q}=a_{q, p}$ that

$$
T_{\mu}\left(a_{p, q}\right)^{t} T_{\mu}=\left(t_{0} / 2 \mu^{3}\right)^{\mu} S_{\mu} .
$$

Here we denoted by $S_{q}$ for $1 \leqq q \leqq \mu, q \times q$ matrix of Jacobi

$$
\left(\begin{array}{llll}
b_{1} & d_{2} & & 0 \\
d_{2} & b_{2} & \ddots & \\
0 & \ddots & \ddots & d_{q} \\
& & d_{q} & b_{q}
\end{array}\right) \quad \begin{aligned}
& b_{1}=4 \mu-1, \\
& b_{q}=-2, \quad d_{q}=1, \\
& q \geqq 2 .
\end{aligned}
$$

If we put $\square_{q}=\operatorname{det} S_{q}$ then we have

$$
\begin{aligned}
\square q & =b_{q} \square d_{q-1}-d_{q}^{2} \square_{q-2} \\
& =-2 \square_{q-1}-\square_{q-2} \quad \text { if } q \geqq 3,
\end{aligned}
$$

which shows

$$
\operatorname{det}\left(a_{p, q}\right)=\left(t_{0} / 2 \mu^{3}\right)^{\mu}\left(1-4 \mu^{2}\right) .
$$

Thus we obtain (4.14)'.

\section{§6. Proof of Proposition 2.2}

It is easy to see that when $\mu \rightarrow \infty$ the trajectory $C_{\mu}$ given in Proposition 2.1 converges the part of $\pi \tilde{C}_{0}$ corresponding to $t \in\left[0, t_{0}\right]$. Then the assumption i) of Proposition 2.2 is obvious. Since it follows from (5.5) that the difference between $\lambda_{1}$ and $\lambda_{2}$ is estimated by $\beta^{4}=\left(t_{0} / 2 \mu\right)^{4}$ on the trajectory $C_{\mu}$ we get (2.2). By means of the argument in the previous section it is not difficult to see the rest of the assertion ii) of Proposition 2.2 because $H_{k}^{j}\left(\lambda_{1}-\lambda_{2}\right)=0(k=1,2, j=0,1,2,3)$ on $\pi \tilde{C}_{0}$.

As stated in Introduction, for the proof of the assertion iii) of Proposition 2.2 we need to review the result of Wakabayashi [11] and [10], where the propagation of $C^{\infty}$ wave front sets and that of wave front sets in Gevrey classes, respectively, were studied for the hyperbolic Cauchy problem. If we apply the result of [11] (resp. [10]) to the Cauchy problem 
for any hyperbolic system of the form (0.1), we get the following description about $C^{\infty}$ wave front set (resp. the one in Gevrey class) of solutions: If $U$ and $G$ denote the solution and the initial value of the Cauchy problem, respectively, then we get

$$
\begin{gathered}
W F U \subset\left\{K_{\bar{\rho}}^{+} ; \tilde{\rho} \in p^{-1}(0) \cap \pi^{-1}(\{0\} \times W F G)\right\}, \\
W F_{\kappa_{1}} U \subset\left\{K_{\bar{\rho}}^{+} ; \tilde{\rho} \in p^{-1}(0) \cap \pi^{-1}\left(\{0\} \times W F_{\kappa_{1}} G\right)\right\} \\
\left(1<\kappa_{1}<2\right),
\end{gathered}
$$

where

$$
p^{-1}(0)=\bigcup_{j=1,2} p_{j}^{-1}(0), \quad p_{j}^{-1}(0)=\left\{\tilde{\rho} \in T^{*}\left(R_{t} \times R_{x}^{n}\right) \backslash 0, p_{j}(\tilde{\rho})=0\right\}
$$

and $K_{\bar{\rho}}^{+}$is defined as follows:

$$
\begin{gathered}
K_{\tilde{\rho}}^{+}=\left\{\tilde{\rho}(s) \in T^{*}\left(R_{t} \times R_{x}^{n}\right) ; s \geqq 0 \text { and } \tilde{\rho}(s)\right. \text { is a Lipshitz } \\
\text { continuous curve satisfying } d \tilde{\rho}(s) / d s \in \Gamma_{\tilde{\rho}}^{o}(s) \text { for } \\
\text { a.e. } s \text { and } \tilde{\rho}(0)=\tilde{\rho}\} \text {. (See (1.2) of [11]). }
\end{gathered}
$$

Here $\Gamma_{\tilde{\rho}}^{\sigma}$ is the set of vector fields in $T^{*}\left(R_{t} \times R_{x}^{n}\right)$ defined by

$$
\Gamma_{\tilde{\rho}}^{\sigma}=\left\{\left(\sum_{j \in \mathscr{S}} \alpha_{j} H_{p_{j}}\right)(\tilde{\rho}) ; \alpha_{j} \geqq 0\right\} \quad \text { if } \tilde{\rho} \in \bigcap_{j \in \mathscr{S}} p_{j}^{-1}(0),
$$

where $\subseteq$ is a subset of $\{1,2\}$.

Noting that $W F U \subset p^{-1}(0) \subset T^{*}\left(R_{t} \times R_{x}^{n}\right) \backslash 0$ we can see that the description (6.1) is equivalent to (0.5) in Introduction because we have the following:

Proposition 6.1. For any closed conic set $\tilde{K} \subset T^{*} R_{x}^{n} \mid 0$ we pet

$$
\Gamma\left(t_{0} ; \tilde{K}\right)=\left\{\left.\pi K_{\bar{\rho}}^{+}\right|_{t=t_{0}} ; \tilde{\rho} \in \pi^{-1}(\{0\} \times \tilde{K}) \cap p^{-1}(0)\right\},
$$

where $\left.\pi K_{\tilde{\rho}}^{+}\right|_{t=t_{0}}$ is the section of $\pi K_{\bar{\rho}}^{+} \subset R_{t} \times T^{*} R_{x}^{n}$ at $t=t_{0}$.

The inclusion relation that the left hand side contains the right hand side was proved in [11] (see Theorem 4.4 of [11]) and another direction of inclusion will be proved in the forthcoming paper [8] by K. Taniguchi and the author.

On account of this proposition, for the proof of the assertion iii) of Proposition 2.2 it suffices to show

$$
B_{\bar{p} 0}=K_{\bar{p} 0}^{+} .
$$


In the similar manner as in the observation of [11] for a simple example of hyperbolic system given by [4] (see Example 4.9 of [11]) we get the following: Since $\Sigma_{1} \equiv p_{1}^{-1}(0) \cap p_{2}^{-1}(0)=\left\{\tilde{\rho} \in T^{*}\left(R_{t} R_{x}^{4}\right) \backslash 0 ; \lambda_{2}(\pi \tilde{\rho})=0\right\}$ we get

$$
T_{\tilde{\rho}} \Sigma_{1} \cap \Gamma_{\bar{\rho}}^{\sigma}= \begin{cases}\Gamma_{\tilde{\rho}}^{\sigma} & \text { if } \partial_{t} \lambda_{2}(\pi \tilde{\rho})=0 \\ \{0\} & \text { otherwise }\end{cases}
$$

because it follows from $\left(\alpha_{1} H_{p_{1}}+\alpha_{2} H_{p_{2}}\right)(\tilde{\rho}) \in T_{\bar{\rho}} \Sigma_{1}$ that $\left(\alpha_{1}+\alpha_{2}\right) \partial_{t} \lambda_{2}(\pi \tilde{\rho})=0$. Put $\Sigma_{2}=\left\{\tilde{\rho} \in \Sigma_{1} ; \partial_{t} \lambda_{2}=0\right\}$. Then we get

$$
T_{\tilde{\rho}} \Sigma_{1} \cap \Gamma_{\tilde{\rho}}^{\sigma}= \begin{cases}\Gamma_{\tilde{\rho}}^{\sigma} & \text { if } t^{2}-4 x_{1} t+8 x_{2}=0 \\ \left\{\alpha\left(H_{p_{1}}+H_{p_{2}}\right)(\tilde{\rho}) ; \alpha \geqq 0\right\} & \text { otherwise },\end{cases}
$$

because it follows from $\left(\alpha_{1} H_{p_{1}}+\alpha_{2} H_{p_{2}}\right)(\tilde{\rho}) \in T_{\bar{\rho}} \Sigma_{2}$ that

$$
\left(\alpha_{1}-\alpha_{2}\right)\left(t^{2}-4 x_{1} t+8 x_{2}\right)=0 .
$$

Putting $\Sigma_{3}=\left\{\tilde{\rho} \in \Sigma_{2} ; t^{2}-4 x_{1} t+8 x_{2}=0\right\}$ we have

$$
T_{\tilde{\rho}} \Sigma_{3} \cap \Gamma_{\tilde{\rho}}^{\sigma}= \begin{cases}\Gamma_{\tilde{\rho}}^{\sigma} & \text { if } t=2 x_{1} \\ \left\{\alpha H_{p_{1}+p_{2}}(\tilde{\rho}) ; \alpha \geqq 0\right\} & \text { otherwise },\end{cases}
$$

because we have $\left(\alpha_{1}-\alpha_{2}\right)\left(t-2 x_{1}\right)=0$ by the same argument. If we put $\Sigma_{4}=\left\{\tilde{\rho} \in \Sigma_{3} ; t=2 x_{1}\right\}$ then we have

$$
T_{\tilde{\rho}} \Sigma_{4} \cap \Gamma_{\tilde{\rho}}^{\sigma}=\left\{\alpha H_{p_{1}+p_{2}}(\tilde{\rho}) ; \alpha \geqq 0\right\} .
$$

In view of (6.6)-(6.9) we obtain (6.5), noting the assertion ii) of Proposition 2.2 .

\section{§7. Proofs of Corollaries 2.4 and 2.5}

If $U$ is the solution of (C.P) it follows from Theorem 5.1 of [1] that

$$
W F_{\kappa} U \subset p^{-1}(0) \equiv \bigcup_{j=1,2} p_{j}^{-1}(0) \quad \text { for } \kappa \geqq 1 .
$$

If $\delta\left(t^{\prime}\right) \in T^{*} R_{x}^{4} \backslash 0$ denotes the end point of $\pi \tilde{C}_{0} \subset R_{t} \times T^{*}\left(R_{x}^{4}\right)$ at $t=t^{\prime}$, for the proof of Corollary 2.4 it suffices to show for any $t^{\prime}$

$$
\delta\left(t^{\prime}\right) \in W F_{\kappa^{\prime}} U\left(t^{\prime}\right) \quad \text { for } \kappa_{0} \leqq \kappa^{\prime}<3
$$

because $\tilde{C}_{0}=\pi^{-1}\left(\pi \tilde{C}_{0}\right) \cap p^{-1}(0)$.

Let $t_{0}$ be a fixed positive and let $G$ be the initial value defined by (3.8) and (3.2). Unfortunately, $G$ depends on $t_{0}$. However, if we choose the sequence $\left\{\tau_{N}\right\}$ suitably we have (7.1) for any $t^{\prime}$ of a countable set 


$$
\left\{t_{j}^{\prime} ; j=1,2, \cdots, t_{j}^{\prime}=\left(r_{j}^{\prime} / r_{j}\right) t_{0}, r_{j}, r_{j}^{\prime}>0 \text { integers }\right\}
$$

Indeed: For $g$ of (3.2) we put

$$
\left\{\begin{array}{l}
g(x)=\Sigma \phi_{N}\left(x, \tau_{N}\right) \\
\phi_{N}(x, \tau)=\tau^{-1} \phi\left(\tau^{1-\gamma} x\right) \exp i \tau x \cdot \eta_{N} \\
\eta_{N} \equiv \eta_{N}\left(t_{0}\right)=\left(-\left(t_{0} / 4 N\right)^{4}, 0,0,1\right) .
\end{array}\right.
$$

Note that for a $t^{\prime}=\left(r^{\prime} / r\right) t_{0}\left(r, r^{\prime}>0\right.$ integers $)$ we have $\eta_{N}\left(t_{0}\right)=\eta_{\left(N r^{\prime} / r\right)}\left(t^{\prime}\right)$. Put $\tilde{N}=N r^{\prime} / r$ for $N=r k(k=1,2, \cdots)$. Let $U^{\tilde{N}}={ }^{t}\left(u_{1}^{\tilde{N}}, u_{2}^{\tilde{N}}\right)$ denote the solution of (C.P) for the initial value

$$
{ }^{t}\left(\psi_{0}(x) \phi_{N}\left(x, \tau_{N}\right), \psi_{0}(x) \phi_{N}\left(x, \tau_{N}\right)\right) \quad \text { with } N=\tilde{N} r / r^{\prime},
$$

where $\psi_{0}(x)$ is the cut function given in (3.8). If $\left(x_{\tilde{N}}, \xi_{\tilde{N}}\right)$ is the end point of admissible trajectories issuing from $\left(0, \eta_{\tilde{N}}\right)\left(=\left(0, \eta_{N}\right)\right)$ of step $2 \tilde{N}$, then by the same way as in the proof of (3.11) we see that there exists a constant $c_{3}>0$ independent of $\tilde{N}$ such that

$$
\left|\left(\widehat{h_{\tilde{N}}} u_{1}^{\tilde{N}}\right)\left(t^{\prime} ; \tau \xi_{\tilde{N}}\right)\right| \geqq c_{3}^{\tilde{N}+1} \tilde{N}^{3 \tilde{N}} \tau^{-\tilde{N}+4(\gamma-1)-1} \quad \text { if } \tau \geqq \tau\left(\tilde{N}, t^{\prime}\right),
$$

where $\tau\left(\tilde{N}, t^{\prime}\right)$ is a large number determined in the same manner as for $\tau(2 N)$ of (4.8) and (4.9). Recall that we have (4.6) only if $\left|\tau_{N}-\tau_{N^{\prime}}\right|$ for $N \neq N^{\prime}$ is large enough to hold (4.4). If the sequence $\left\{\tau_{N}\right\}$ satisfies

$$
\tau_{N} \geqq \tau\left(\tilde{N}, t^{\prime}\right) \quad \text { for } N=r k(k=1,2,3, \cdots)
$$

then there exists a constant $C_{\tilde{N}}>0$ such that

$$
\left|\left(\widehat{h_{N} u_{1}}\right)\left(t^{\prime} ; \tau_{N} \xi_{\tilde{N}}\right)\right| \geqq\left(c_{3}^{\tilde{N}+1} \tilde{N}^{3 \tilde{N}}-C_{\tilde{N}} \tau_{N}^{-1}\right) \tau_{N}^{-\tilde{N}+4(\gamma-1)-1},
$$

where $U={ }^{t}\left(u_{1}, u_{2}\right)$ is the solution of (C.P) for the $G$ defined by (3.8) and (3.2). If we assume that $\tau\left(\tilde{N}, t^{\prime}\right)$ is large enough to hold for $\tau_{N}$ satisfying (7.5)

$$
c^{3 \tilde{N}+1} \tilde{N}^{3 \tilde{N}}-C_{\tilde{N}} \tau_{N}^{-1} \geqq c_{3}^{\tilde{N}+1} \tilde{N}^{3 \tilde{N}} / 2
$$

it follows from Corollary 1.3 and its remark that we have (7.1) for $t^{\prime}=$ $\left(r^{\prime} / r\right) t_{0}$. If we choose the sequence $\left\{\tau_{N}\right\}$ such that

$$
\tau_{N} \geqq \max _{1 \leqq j \leqq N} \tau\left(\tilde{N}_{j}, t_{j}^{\prime}\right)
$$

we get (7.1) for any $t^{\prime} \in\left\{t_{j}^{\prime}\right\}_{j=1}^{\infty}$. Here we putted $\tau\left(\tilde{N}_{j}, t_{j}^{\prime}\right)=0$ if $\tilde{N}_{j}=N r_{j}^{\prime} / r_{j}$ is not integer. 
Since $\left\{t_{j}^{\prime}\right\}$ is dense in $R^{+}$we have (7.1) for any $t^{\prime}>0$ because $W F_{\kappa} U$ is closed set in $T^{*}\left(R_{t} \times R_{x}^{4}\right) \backslash 0$. Since we can obtain the same result as Theorem 2.3 for negative $t_{0}$, we get (7.1) for any $t^{\prime}$.

In the rest of this section we shall prove Corollary 2.5. We first remark that if $U$ and $G$ are the solution and the initial value, respectively, of the Cauchy problem for any hyperbolic system of the form (0.1) then it follows that for any $\kappa>1$

$$
W F_{\kappa} U\left(t_{0}\right) \subset \Gamma\left(t_{0} ; W F_{\kappa} G\right), \quad t_{0}>0 .
$$

In view of Proposition 6.1 the inclusion with $1<\kappa<2$ follows from (6.1)' The case for $\kappa \geqq 2$ will be proved in [8].

For a fixed $t_{0}>0$ let $t^{\prime}$ be a positive such that $0<t^{\prime}<t_{0}$. Let $\tilde{C}_{1}$ be the continuous curve in $T^{*}\left(R_{t} \times R_{x}^{4}\right)$ such that

$$
\tilde{C}_{1}=\left\{\begin{array}{l}
\tilde{C}_{0} \quad \text { for } 0 \leqq t \leqq t^{\prime} \\
\text { Hamilton flow by } H_{p_{j}} \quad(j=1,2) \text { for } t^{\prime} \leqq t \leqq t_{0} .
\end{array}\right.
$$

If $\tilde{\delta} \in T^{*} R_{x}^{4} \backslash 0$ denotes the end point at $t=t_{0}$ of the curve $\pi \tilde{C}_{1}$ then it follows that

$$
\tilde{\delta} \in W F_{\kappa^{\prime}} U\left(t_{0}\right), \quad \kappa_{0} \leqq \kappa^{\prime}<3 .
$$

For the proof of (7.7) it suffices to consider the sequence of admissible trajectories issuing from $\rho_{\mu}$ which converges the curve $\pi \tilde{C}_{1}$ and to find an initial value $G$ corresponding to the sequence of admissible trajectories, as in the proof of Theorem 2.3. Though the initial value $G$ depends on $\tilde{\delta} \in \Gamma\left(t_{0} ; \tilde{K}\right)$ we can complete the proof of Corollary 2.5 by the same argument as in the proof of Corollary 2.4.

Acknowledgement. The author wishes to thank Professor J. Sjöstrand for suggesting the use of $\phi$ in (3.2), which permitted us to ameliorate the primitive result. The main part of this study was obtained when the author stayed at Orsay in 1981-3, supported by the scholarship of French Government.

\section{REFERENCES}

[1] L. Hörmander, Uniqueness theorems and wave front sets for solutions of linear differential equations with analytic coefficients, Comm. Pure Appl. Math., 24 (1971), 671-704.

[2] - Spectral analysis of singularities, Seminar of Singularities of Solutions of Linear Partial Differential Equations, Princeton University Press 1979, 3-49. 
[ 3 ] V. Ya. Ivrii, Wave fronts of solutions for symmetric pseudodifferential systems, Sibirsk. Mat. Z., 20 (1979), 557-578.

[ 4 ] C. Iwasaki and Y. Morimoto, Propagation of singularities of solutions for a hyperbolic system with nilpotent characteristics, I, Comm. Partial Differential Equations, 7 (1982), 743-794.

[5] — - Propagation of singularities of solutions for a hyperbolic system with nilpotent characteristics, II, Comm. Partial Differential Equations, 9 (1984), 14071436.

[6] — Precise propagation of singularities for a hyperbolic system with characteristics of variable multiplicity, Nagoya Math. J., 101 (1986), 111-130.

[ 7 ] H. Kumano-go and K. Taniguchi, Fourier integral operators of multi-phase and the fundamental solution for a hyperbolic equation, Funkcial. Ekvac., 22 (1979), 161-196.

[ 8 ] Y. Morimoto and K. Taniguchi, Propagation of wave front sets of solutions of the Cauchy problem for hyperbolic equations in Gevrey classes, to appear.

[9] F. Treves, Introduction to pseudo-differential and Fourier integral operators, Vol. 1, Plenum Press (1981).

[10] S. Wakabayashi, Singularities of solutions of the Cauchy problem for hyperbolic systems in Gevrey classes, Japan. J. Math., 11 (1985), 157-201.

[11] - Singularities of solutions of the Cauchy probiem for symmetric hyperbolic systems, Comm. Partial Differential Equations, 9 (1984), 1147-1177.

Department of Engineering

Mathematics, Nagoya University

Chikusa-ku, Nagoya 464, Japan 